\title{
Dissectional investigation: variations in morphology of the teres minor tendon and muscle-tendon junction
}

\author{
Samuel L Holley,2, Spencer L Hill ${ }^{1,2}$, Taylor D Ottesen ${ }^{2,3}$, Paul K Vance ${ }^{2,4}$, Lucky Challyandra ${ }^{5}$, Aaron Z Katrikh ${ }^{5}$, David C Whiting ${ }^{6}$, M
} Elena Stark ${ }^{5}$ and Jonathan J Wisco ${ }^{2,7,8^{*}}$

${ }^{1}$ Rocky Vista University College of Osteopathic Medicine, USA

${ }^{2}$ Department of Physiology and Developmental Biology, Brigham Young University, USA

${ }^{3}$ Yale School of Medicine, USA

${ }^{4}$ Pacific Northwest University of Health Sciences, College of Osteopathic Medicine

${ }^{5}$ Division of Integrative Anatomy, Department of Pathology and Laboratory Medicine, David Geffen School of Medicine, USA

${ }^{6}$ Department of Statistics, Utah Valley University, USA

${ }^{7}$ Department of Neurobiology and Anatomy, University of Utah Medical School, USA

${ }^{8}$ Department of Anatomy and Neurobiology, Boston University School of Medicine, USA

\section{Introduction}

The teres minor (TM) muscle is a fusiform muscle that originates on the lateral border and adjacent posterior surface of the scapula and inserts onto the posterior aspect of the greater tubercle of the humerus and posterior joint capsule. As a member of the rotator cuff, the TM externally rotates the humerus and modulates the action of the deltoid muscle, preventing the humeral head from sliding superiorly during abduction.

Before 2016, investigation of the TM muscle was limited to the morphology of its fascial covering and nervous supply. The TM is innervated by the posterior branch of the axillary nerve and has less innervation variability than the supraspinatus, as found by Bacle, et al. [1]. They also recently demonstrated anatomical variations of the fascial coverings but did not describe the TM muscle-tendon junction of humerus [2]. Other studies have shown, through magnetic resonance imaging (MRI) that in rotator cuff tears involving the infraspinatus muscle, the TM can hypertrophy and provide compensation [3]. Kikukawa and associates investigated the benefits of hypertrophy of TM muscles for posterosuperior-rotator cuff tears. They confirmed that the hypertrophy leads to an increase in external rotation of the shoulder and can play an important clinical role in orthopedics and sports medicine [4]. However, there remains a paucity of research examining the muscle-tendon junction of the TM. New information on the variations in morphology of the muscle-tendon junction and nervous insertion may prove impactful on presurgical planning and surgical outcomes.

To investigate this potential presurgical tool, variation in the TM muscle-tendon junction was noted through dissection of human cadaver specimens. Frequency of variation in muscle-tendon junction, tendon length, and tendon width of each muscle-tendon junction variation was noted. Additionally, the muscle innervation of many specimens was noted. Through the utilization of human cadavers, the current study investigates the incidence of variation of the TM muscletendon junction and possible applicability to presurgical planning to improve outcomes.

\section{Methods}

The TM muscle was dissected in 41 formalin-embalmed specimens obtained from Brigham Young University (BYU), Rocky Vista University College of Osteopathic Medicine (RVUCOM), and UCLA David Geffen School of Medicine (DGSOM) Donated Body Programs. Appropriate research consent and approval was obtained from each institution, and each cadaver ID number was recorded. The study was exempt from IRB approval. Dissection teams were led by S.L. Holley, who managed the procedure described below in identifying and preserving the TM muscle and axillary nerve. Our UCLA DGSOM dissection team consisted of M.E. Stark, L. Challyandra, and A.Z. Katrikh. The RVUCOM dissection was performed by S.L. Holley and S.L. Hill, while S.L. Holley isolated the TM with P.K. Vance at BYU. Information about the cadavers was limited as different institutions didn't provide the same data, for example age, dominance, and medical history for each set of cadavers. Cadaveric shoulders that were not intact and had already been dissected by medical students for their curriculum were excluded.

The cadavers were placed in a prone position where the skin and subcutaneous tissue of the back and shoulder were removed to expose the muscles arising from the posterior aspect of the scapula. The trapezius muscle was incised along the medial attachments to the spinous processes of the vertebral column and the superolateral attachments on the scapula and clavicle. The trapezius muscle was reflected superiorly to better expose the posterior aspect of the deltoid muscle and the rotator cuff muscles. The deltoid muscle was incised along the attachments to the clavicle, as well as the spine and acromion

*Correspondence to: Jonathan J Wisco, Ph.D., Associate Professor, Boston University School of Medicine, Department of Anatomy and Neurobiology, Laboratory for Translational Anatomy of Degenerative Diseases and Developmental Disorders (TAD4), 72 E Concord St, L-1004, Boston, MA 02118, Tel: 310-746-6647, 617-358-3546; E-mail: jjwisco@bu.edu

Key words: Teres minor, tendon insertion pattern

Received: December 27, 2019; Accepted: January 20, 2020; Published: January 23,2020 
of the scapula. The deltoid muscle was reflected laterally to expose the head of the humerus, and the distal attachments of the rotator cuff muscles. The axillary nerve and accompanying posterior circumflex humeral vasculature along the deep surface of the deltoid was preserved. The fascia overlying the TM and infraspinatus muscles was removed with blunt dissection to clean the surface of the muscles in order to visualize the attachment of the TM. Because the TM is closely related to the infraspinatus, care was taken to identify the TM by the insertion and innervation. Probe dissection was used to further separate the TM from the infraspinatus muscle.

Measurements $(\mathrm{cm})$ of the tendon width were taken using a flexible ruler at the widest point of the tendon. Measurements and photographs were taken of the length of the TM tendon from the most proximal point of the muscle-tendon junction to the distal insertion point on the humerus. The appearance of the TM muscle-tendon junction was noted as either single-headed (Non-Classic) or two-headed (Classic). The distal insertion point of the tendon was recorded as well. Standard error of the mean and T-test was calculated for each set of measurements including length, width, and nerve penetration for each classic and non-classic morphology.

\section{Results}

Upon dissection of 43 specimens, 35 (85\%) were classified as "Classic" (Figure 1a) muscle-tendon junction morphology and 6 (15\%) as "Non-Classic" (Figure 1b) muscle-tendon junction morphology. Two of the specimens (5\%) were unidentifiable. The average length of the tendon was $37.9 \mathrm{~mm}$ (SEM $1.39 \mathrm{~mm}$ ) and $49.8 \mathrm{~mm}$ (SEM $4.24 \mathrm{~mm}$ ) for classic and non-classic respectively. The average width was $15.3 \mathrm{~mm}$ (SEM $0.85 \mathrm{~mm}$ ) and $19.7 \mathrm{~mm}$ (SEM $2.19 \mathrm{~mm}$ ) for classic and non-classic respectively.

Measurements were taken of the distance from TM tendon insertion to the axillary nerve insertion into the TM muscle on 21 specimens. The average distance for classic was $52.4 \mathrm{~mm}$ (SEM $2.37 \mathrm{~mm}$ ) and nonclassic was $57.3 \mathrm{~mm}$ (SEM $4.39 \mathrm{~mm}$ ). There were no notable differences in location of TM insertion into the humerus for either classic or nonclassic morphology. The average distance of the 21 specimens was $53.3 \mathrm{~mm}$ (SEM $2.09 \mathrm{~mm}$ ).

The boxplots and the summary statistics in Figure 2 give reason to believe that the non-classic measurements are greater than the classic measurements. Three hypothesis tests were performed below using Welch's T-test with the assumption of normality and nonequal variances. With using $\alpha=0.1$ we found significance in 2 of the 3 tests we did, reported below. We saw the tendon length for nonclassic is significantly greater than the classic $(\mathrm{P}$-value $=0.01794)$. The non-classic tendon width was significantly greater than the classic. (P-value $=0.05381)$. The nerve attachment distance was not significantly different between non-classic and classic morphologies (P-value=0.1859).
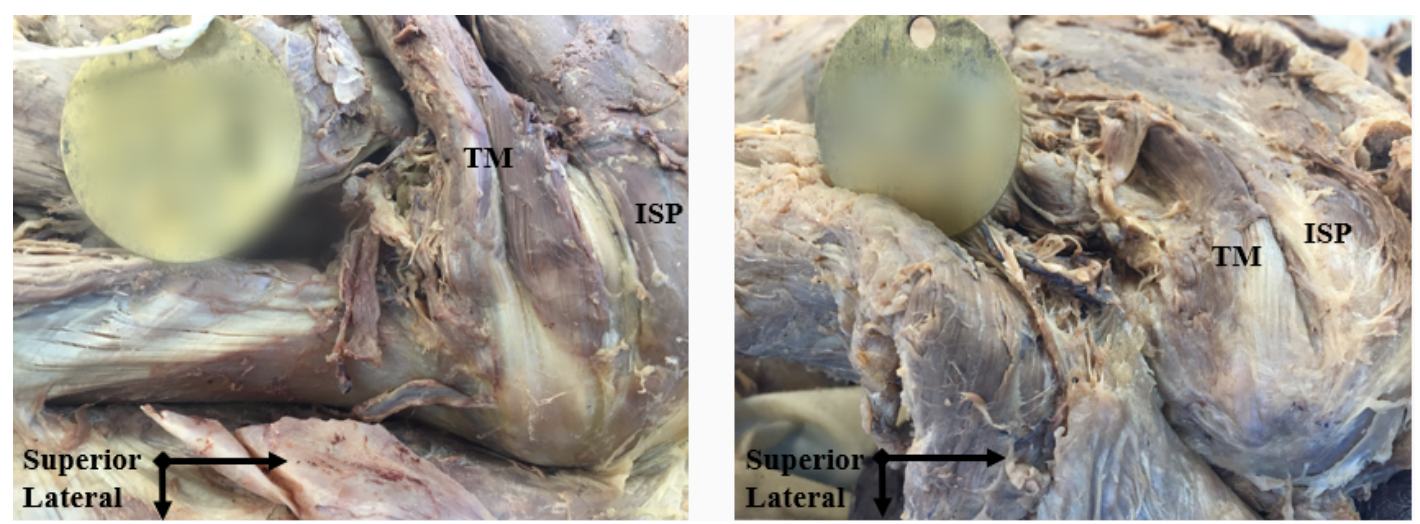

Figure 1. Figure 1a: Two-headed muscle tendon morphology or "Classic"; Figure 1b: One-headed muscle-tendon junction morphology or "Non-Classic"

2a Tendon Length

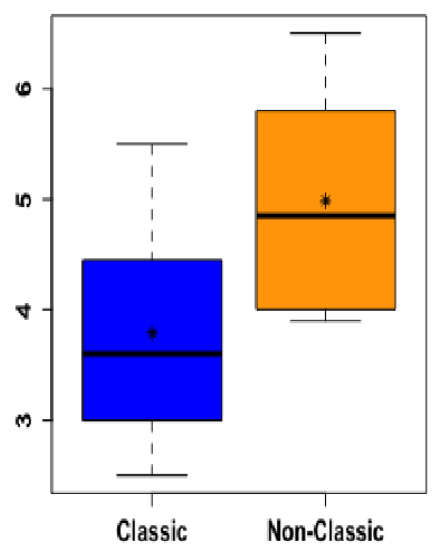

$2 b$ Tendon Width

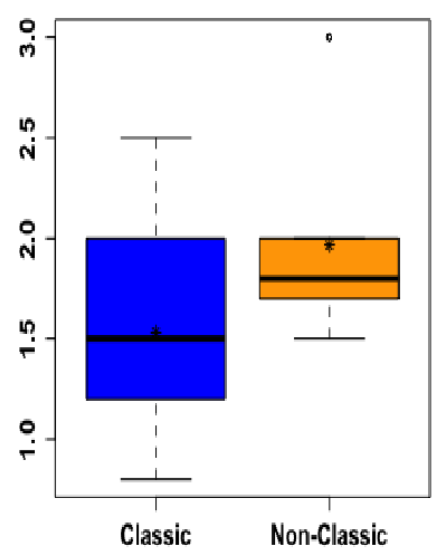

\section{2c Nerve Attachment Distance}

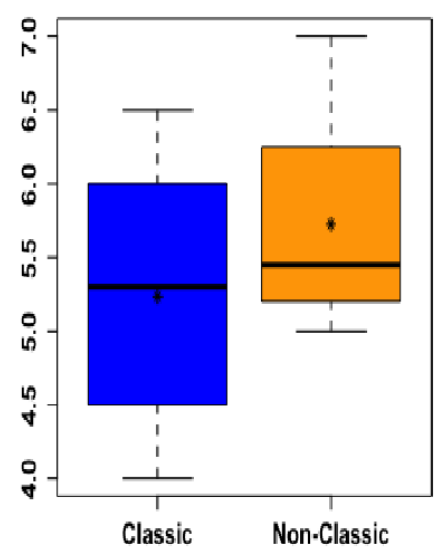

Figure 2. "Asterisks in box plots represent the mean. 2a; Tendon length $(\mathrm{cm})$, Non-classic $>$ classic, $\mathrm{p}$-value $=0.01794,90 \%$ CL. 2b; Tendon width $(\mathrm{cm})$, Non-classic $>$ classic, p-value $=0.5381$, $90 \%$ CL; Nerve attachment distance $(\mathrm{cm})$, Non-classic not $>$ classic, p-value $=0.1859,90 \% \mathrm{CL}$ 


\section{Discussion}

The current study describes two different patterns of morphology associated with the muscle-tendon junction of the TM muscle. The first pattern (Figure 1a) designated as classic was on average $11.9 \mathrm{~mm}$ shorter, $4.4 \mathrm{~mm}$ narrower, had $4.9 \mathrm{~mm}$ shorter nerve penetration, two heads and was more common with $81 \%$ of cadavers exhibiting this morphology. The second pattern (Figure 1b) designated as non-classic was longer, wider, had longer nerve penetration, one head, and was found in $14 \%$ of cadavers. $5 \%$ of specimens were non-identifiable due to the extended use of the specimen by the institution.

Current literature regarding the TM is limited to anatomical variants of fascial coverings and nervous supply and does not describe the muscle tendon-junction [2]. Explanation of different morphologies of the muscle-tendon junction and their applicability is currently unknown. The authors postulate that the different morphologies arose from an embryologic origin, a hypertrophied TM secondarily to a rotator cuff tear, and/or from atrophy of the classic variant resulting in a non-classic presentation. Further research investigating possible reasons and other potential morphologies may provide critical insight.

Clinically, the TM plays an important anatomic role by creating the superior border of the quadrangular space. Both the axillary nerve and posterior circumflex humeral artery pass from the anterior axilla to the posterior axilla through the quadrangular space creating potential for compression or damage during trauma or deterioration. As a result, hypertrophy of the TM muscle has been shown to play an important supportive role following rotator cuff injuries [3].

Surgical and functional outcomes have also been shown to be affected by the integrity of the TM muscle both pre- and postoperatively [3]. Presence of atrophy and/or severe fatty infiltration on magnetic resonance imaging (MRI) of the TM muscle was shown in several studies to be a prognostic factor of decreased active elevation and external rotation following reverse total shoulder arthroplasty [57]. Similarly, patients with a severely atrophied TM muscle on MRI or conventional diagnostic plain-film x-ray imaging exhibited lesser external rotation and Constant score than moderately atrophied or intact TM muscle counterparts [8]. Additionally, studies found fatty infiltration of the TM on MRI to be predictive of outcomes following latissimus dorsi tendon transfers for those with irreparable rotator cuff repairs $[9,10]$.

The results of these studies suggest the condition of the TM muscle prior to surgical intervention is an important indicator of postoperative outcomes and should be a consideration prior to treatment [4]. Further, the previously mentioned studies suggest an increasing support of the importance of the TM as a factor for recovery from a reverse shoulder arthroplasty and other surgical procedures. Although these studies describe the correlation of atrophy and fatty infiltration of the TM with outcomes, the present introductory study describes an important new consideration for understanding a patient's recovery course based on TM muscle health - morphology.

Variations in the TM muscle morphology may influence the TM muscle condition following trauma such as a major rotator cuff tear. Knowledge of what type of TM pattern a patient presents with may influence what outcomes can be expected and could be an important factor for physicians to more accurately design patient treatment plans, describe recovery times, and explore physical therapy options. For example, knowledge of morphology differences may indicate an increased benefit of strengthening exercises for a patient prior to surgery when compared to cohorts of a different morphological construct. These personalized treatment adaptations may increase recovery time and functional outcomes following corrective procedures such as reverse shoulder arthroplasty. Additional studies to compare differences in outcomes for patients with different morphological variants and arthroplasty approaches are merited.

The current study identifies potentially important differences in morphology of the TM muscle. Through preoperative imaging, the morphology of a patient's TM can be assessed, and surgical recommendations adapted based on results. The authors understand the need of further studies with MRI and or US to see the full utility of TM morphology and its potential for better pre-surgical planning to maximize post-surgical outcomes. This is a step towards utilizing imaging more precisely to create more personalized medicine in orthopedics.

\section{Acknowledgements}

The authors wish to thank individuals who donate their bodies and tissues for the advancement of education and research.

\section{References}

1. Bardeen CR, Evans HM, Felix W, Keibel F, Lewis FT, et al. Manual of human embryology 12th ed; The Muscular System. Philadelphia \& London: J.B. Lippincott Company.

2. Bacle G, Gregoire JM, Patat F, Clavert P, de Pinieux G, et al. (2017) Anatomy and relations of the infraspinatus and the teres minor muscles: a fresh cadaver dissection study. Surg Radiol Anat 39:119-26. [Crossref]

3. Kikukawa K, Ide J, Kikuchi K, Morita M, Mizuta H, et al. (2014) Hypertrophic changes of the teres minor muscle in rotator cuff tears: quantitative evaluation by magnetic resonance imaging. J Shoulder Elbow Surg 23: 1800-18005. [Crossref]

4. Kikukawa K, Ide J, Terakawa Y, Takada K, Morita M, et al. (2016) Hypertrophic teres minor restores shoulder strength and range of external rotation in posterosuperior rotator cuff tears. J Shoulder Elbow Surg 25: 1882-1888. [Crossref]

5. Boileau P, Watkinson D, Hatzidakis AM, Hovorka I (2006) Neer Award 2005: The Grammont reverse shoulder prosthesis: Results in cuff tear arthritis, fracture sequelae, and revision arthroplasty. J Shoulder Elbow Surg 15: 527-540. [Crossref]

6. Simovitch RW, Helmy N, Zumstein MA, Gerber C (2007) Impact of fatty infiltration of the teres minor muscle on the outcome of reverse total shoulder arthroplasty. $J$ Bone Joint Surg Am 89:934-939. [Crossref]

7. Sirveaux F, Favard L, Oudet D, Huquet D, Walch G, et al. (2004) Grammont inverted total shoulder arthroplasty in the treatment of glenohumeral osteoarthritis with massive rupture of the cuff. Results of a multicentre study of 80 shoulders. J Bone Joint Surg $\operatorname{Br}$ 86:388-395. [Crossref]

8. Pape G, Bruckner T, Loew M, Zeifang F (2013) Treatment of severe cuff tear arthropathy with the humeral head resurfacing arthroplasty: two-year minimum followup. J Shoulder Elbow Surg 22: e1-7. [Crossref]

9. Costouros JG, Espinosa N, Schmid MR, Gerber C (2007) Teres minor integrity predicts outcome of latissimus dorsi tendon transfer for irreparable rotator cuff tears. J Shoulder Elbow Surg 16: 727-734. [Crossref]

10. Nove-Josserand L, Costa P, Liotard JP, Safar JF, Walch G, et al. (2009) Results of latissimus dorsi tendon transfer for irreparable cuff tears. Orthop Traumatol Surg Res 95: 108-113. [Crossref]

Copyright: (C2020 Holley SL. This is an open-access article distributed under the terms of the Creative Commons Attribution License, which permits unrestricted use, distribution, and reproduction in any medium, provided the original author and source are credited. 